\title{
Estudios etiológicos de la marchitez del aguacate en Antioquia-Colombia ${ }^{1}$
}

\author{
Joaquín Guillermo Ramírez Gil², Darío Antonio Castañeda Sánchezz, Juan Gonzalo Morales Osorio ${ }^{4}$
}

\section{RESUMEN}

El cultivo de aguacate en Colombia ha presentado un incremento en el área sembrada durante la última década, especialmente en clima frío moderado, entre 1.800 y $2.500 \mathrm{msnm}$. La variedad Hass se destaca por tener la mayor área sembrada en este clima, en el departamento de Antioquia-Colombia. Este cultivo presenta grandes retos tecnológicos para su expansión; entre ellos el manejo de enfermedades ocupa un lugar predominante por las implicaciones que tiene en costos, impacto en la salud, ambiente y restricciones para exportaciones. La marchitez, ocasiona la muerte de numerosos árboles en todas las etapas del cultivo y presenta la mayor incidencia y severidad de las enfermedades identificadas para este cultivo. El desconocimiento de los agentes causales de esta enfermedad, ha llevado a realizar prácticas de manejo encaminadas principalmente al control del oomycete Phytophthora cinnamomi Rands, como único agente causal. Esta investigación tuvo como objetivo identificar los distintos agentes causales de la marchitez del aguacate y la relación de su incidencia con las prácticas de manejo en lotes cultivados en el departamento de Antioquia-Colombia. Los resultados encontrados muestran que la marchitez es el principal problema del cultivo de aguacate. Los microorganismos P. cinnamomi, Phytophthora heveae Thompson, Phytophthora citrícola Sawada, Verticillium sp., y Cylindrocarpon destructans (Zinss) Scholten fueron aislados y reprodujeron los síntomas asociados a marchitez. Las condiciones de bajo contenido de oxigeno el suelo también reprodujeron la sintomatología de marchitez, involucrando también un origen abiótico en la etiología.

Palabras clave: variedad Hass, enfermedades, Persea americana Mill.

\section{RESUMO}

\section{Estudos etiológicos da murcha-do-abacateiro em Antioquia, Colômbia}

O plantio de abacateiro na Colômbia tem apresentado aumento da área cultivada durante a última década, especialmente em clima frio moderado, com altitudes entre 1.800 e 2.500 m. A variedade Hass destaca-se por apresentar a maior área cultivada nesse clima, no Estado de Antioquia - Colômbia. A cultura do abacateiro apresenta grandes desafios tecnológicos para sua expansão na Colômbia, sendo o manejo das doenças, especialmente da murcha, um dos mais importantes fatores a serem considerados, devido ao impacto nos custos, na saúde, no ambiente e nas restrições para as exportações. $\mathrm{O}$ murchamento ocasiona a morte de muitas árvores em todas as etapas do cultivo, e apresenta a maior incidência e severidade em relação a outras doenças para esse tipo de cultivo. O desconhecimento dos fatores que geram essa doença tem levado à realização de práticas de manejo direcionadas principalmente ao controle do oomiceto Phytophthora cinnamomi Rands como único agente causal. Esta pesquisa teve como objetivo principal identificar possíveis agentes causais da murcha-do-abacateiro na Colômbia, bem como correlacionar a incidência da doença às práticas de manejo

Enviado el 19/10/2012 y aprobado el 04/09/2013.

'Este trabajo hace parte de la Tesis de Maestría en Ciencias Agrarias del primer autor.

${ }^{2}$ Ingeniero Agrónomo, Magíster en Ciencias Agrarias. Universidad Nacional de Colombia, Sede Medellín, Departamento de Ciencias Agronómicas, Campus El Volador, Bloque 11, A.A, 1779, Medellín, Colombia. jgramireg@unal.edu.co (autor para correspondencia).

${ }_{3}^{3}$ Ingeniero Agrónomo, PhD. Universidad Nacional de Colombia sede Medellín. Departamento de Ciencias Agronómicas, Medellín, Colombia. dacastanedas@gmail.com

${ }^{4}$ Ingeniero Agrónomo, PhD. Universidad Nacional de Colombia sede Medellín. Departamento de Ciencias Agronómicas, Medellín, Colombia. jgmoraleso@unal.edu.co 
associadas a essa doença no Estado de Antioquia - Colômbia. Os resultados encontrados mostram que a murcha é o principal problema nos cultivos do abacateiro. Os microorganismos P. cinnamomi, Phytophthora heveae Thompson, Phytophthora citrícola Sawada, Verticillium sp.e Cylindrocarpon destructans (Zinss) Scholte isolados de abacateiros com murcha reproduziram os sintomas associados a essa doença. Deficiências de oxigênio também reproduziram os sintomas da murcha, indicando envolvimento de fatores abióticos na etiologia da murcha-do-abacateiro.

Palavras-chave: variedade Hass, doenças, Persea americana Mill.

\section{INTRODUCCIÓN}

El aguacate es una fruta que tiene un alto potencial, gracias a su aceptado consumo en fresco y las cualidades que tiene para su procesamiento agroindustrial. Actualmente se cultiva en 59 países tropicales y regiones sub-tropicales (Bernal \& Cipriano, 2008). México ocupa el primer lugar en plantaciones comerciales con $49 \%$ del área total cultivada en el mundo, seguido de Colombia con el $13 \%$, posterior se encuentran Chile con $10 \%$ y Estados Unidos con 7\%; otros países productores son Sud África, Israel, Perú, Australia, España, Kenia, entre otros, los cuales suman el 21\% restante (Guerrero et al., 2011; Mejía, 2010).

El cultivo de aguacate en Colombia se desarrolla desde el nivel del mar hasta los 2.500 metros de altura, distribuido en 18 departamentos, donde su producción es destinada principalmente para el mercado local (Bernal \& Cipriano, 2008; Ríos \& Tafur, 2003). Este sistema productivo se ha caracterizado por la siembra de árboles nativos principalmente. El aumento del consumo per capita interno y su potencial exportador como fruta fresca y procesada, ha impulsado el área sembrada de variedades mejoradas aptas para varios climas, destacándose la variedad Hass para clima frio moderado. La mayor área sembrada de esta variedad se encuentra en el departamento de Antioquia con 2.300 ha y con una proyección de 10.000 ha al 2015 (Ruiz, 2010; Mejía, 2010; Bernal \& Cipriano, 2008; Ríos \& Tafur, 2003).

El aumento rápido del área sembrada plantea grandes retos tecnológicos para garantizar la sostenibilidad de las plantaciones. El cultivo del aguacate presenta un número importante de problemas fitosanitarios dentro de los cuales se destacan por su importancia las enfermedades de la raíz. Las raíces y el sistema vascular de los árboles son afectados por diversos microorganismos, causando síntomas similares lo que dificulta su diagnóstico y correcto manejo. Como el patógeno más limitante del cultivo se ha reportado al oomycete Phytophthora cinnamomi Rands, el cual puede causar pérdidas hasta del 90\% (Pérez, 2008; Tamayo, 2007; Ciro et al., 2006; Coffey, 1991; Zentmyer, 1984). Otros patógenos encontrados afectando raíces y tallo han sido: Phytophthora heveae Thompson, Phytophthora citrícola Sawada, Verticillium sp., Armillaria mellea (Vahl: Fr.) Kumm, Cylindrocladium sp., Rosellinia sp., Fusarium solani Sacc, Fusarium oxysporum Schlecht, Fusarium equiseti (Corda) Sacc. sensu Gordon, Rhizoctonia sp., Phymatotrichum omnivorum (Shear) Duggar, Cylindrocladiella sp., Cylindrocarpon sp., Phytium sp. y los nematodos Helicotylenchus sp., Rotylenchulus sp. y Pratylenchus sp. (Vitale et al., 2012; Dann et al., 2011; Bonilla et al., 2011; Pérez, 2008; Tamayo, 2007; Besoain \& Piontelli, 1999; Zentmyer, 1984).

Estos patógenos pueden afectar la planta en todos sus estados de desarrollo induciendo síntomas similares, caracterizados por marchitez generalizada, estancamiento del desarrollo, pérdida de vigor, color, brillo y amarillamiento de las hojas y finalmente en estados avanzados, muerte de los árboles (Pérez, 2008; Tamayo, 2007; Zentmyer, 1984). Coincidiendo con la sintomatología mencionada anteriormente para las enfermedades de origen biótico en aguacate, se reporta a los problemas de déficit de oxígeno en el suelo, como una de las causas más frecuentes de la muerte en campo, consecuencia directa de la siembra en suelos inundados o con problemas de drenaje. (Duque, 2011; Schaffer, 2006; Stolzy et al., 1967).

En Colombia, asociados al complejo marchitez se han reportado a $P$. cinnamomi, Verticillium sp., A. mellea, Cylindrocladium sp., Rosellinia sp., Fusarium sp., Rhizoctonia sp, los nemátodos Helicotylenchus sp., Rotylenchulus sp. y Pratylenchus sp. (Tamayo, 2007; Ciro et al., 2006; Buriticá, 1999). Además de problemas asociados con la asfixia radicular, consecuencia de los bajos niveles de oxígeno en el suelo (Duque, 2011). En el país estos agentes causales han sido poco estudiados y su impacto en los cultivos establecidos es prácticamente 
desconocido. Duque (2011) y Tamayo (2007) coinciden en que solo durante el establecimiento de las plantaciones se generan pérdidas entre 30 y $50 \%$ de las plántulas sembradas. Atendiendo a esta problemática este trabajo tuvo como objetivo identificar los distintos agentes causales de la marchitez del aguacate y la relación de su incidencia con las prácticas de manejo en lotes cultivados en el departamento de Antioquia-Colombia.

\section{MATERIALES Y MÉTODOS}

\section{Localización}

El estudio se realizó en los municipios de Donmatías, Entreríos y San Pedro de Los Milagros ubicados en el Altiplano Norte de Antioquia, Colombia. La región analizada se ubica en una zona de clima frío moderado, en alturas entre 2.200 y $2.550 \mathrm{msnm}$. Se seleccionaron 4 lotes en Donmatías: DL1 (400 plantas), DL2 (500 plantas), DL3 (500 plantas) y DL4 (300 plantas); 3 en Entreríos: EL1 (450 plantas), EL2 (250 plantas) y EL3 (300 plantas) y 3 en San Pedro de Los Milagros: SPL1 (450 plantas), SPL2 (485 plantas) y SPL3 (250 plantas), para un total de 10 lotes con un área de 23 hectáreas, en las cuales se identificaron las variedades Hass (80\%), Reed (15\%), Fuerte (4\%) y Choquette (1\%), las cuales son susceptibles a la marchitez (Figura 1).

\section{Incidencia de la marchitez y de los agentes causales implicados}

La incidencia de la marchitez del aguacate se calculó como el número de plantas enfermas sobre el número total de árboles plantados en cada lote. Para los agentes causales implicados la incidencia se calculó con base en la cantidad de muestras positivas sobre las 15 muestras sintomáticas colectadas en campo.

\section{Relación entre variables de manejo agronómico $e$ incidencia de la marchitez.}

En cada finca/lote se realizó una encuesta sobre las labores desarrolladas en el cultivo y las medidas adoptadas para el manejo de la marchitez. Los porcentajes de incidencia de la marchitez registrados, se transformaron logarítmicamente para su normalización. Luego se analizaron mediante la prueba de rangos múltiples de Tukey $(P<0.05)$. Las variables de manejo agronómico se agruparon en dos categorías, en la primera se incluyeron las variables asociadas al cultivo y en la segunda categoría, se agruparon las variables relacionadas con el manejo fitosanitario de la marchitez. Se estableció una relación de cada uno de estos grupos de variables con la incidencia de la enfermedad mediante un modelo lineal generalizado, usando una función de distribución de Poisson; los cálculos se realizaron mediante el programa

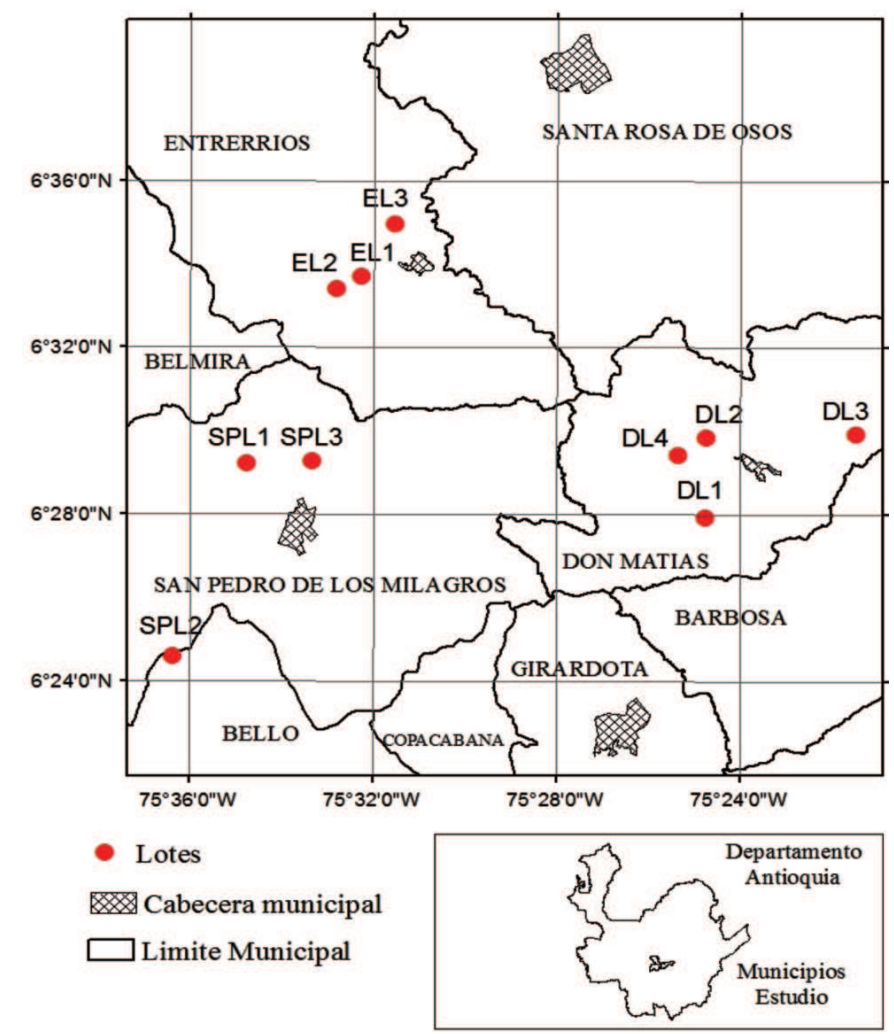

Figura 1. Mapa de ubicación de los lotes muestreados en el departamento de Antioquia-Colombia. Lotes: Donmatías: DL1, DL2, DL3 y DL4; Entreríos: EL1, EL2 y EL3 y San Pedro de Los Milagros: SPL1, SPL2 y SPL3. 
computacional R (Schabenberger \& Pierce, 2002), las variables que no fueron estadísticamente significativas $(P<0.05)$, se eliminaron del modelo. Se construyó un segundo modelo lineal generalizado con las variables restantes y sin incluir las variables que resultaron significativas en el primero, con el objeto de analizar la significancia de variables importantes, las cuales podrían aparecer enmascaradas y que podrían ser determinantes para la incidencia de la enfermedad.

\section{Toma de muestras en campo}

Para la identificación de microorganismos asociados a los síntomas, se colectaron muestras de 15 plantas con sintomatología típica en cada lote analizado. Para cada muestra se realizaron 3 perforaciones en el suelo de la zona radicular, colectando raíces $(20 \mathrm{~g})$ y suelo $(500 \mathrm{~g})$ hasta $30 \mathrm{~cm}$ de profundidad, adicionalmente se tomaron tejidos de la parte basal del tallo (50 g). Las muestras de tejido vegetal se colocaron en servilletas de papel y se transportaron en bolsas plásticas hasta el laboratorio de Estudios moleculares en Ciencias Agrarias y Sanidad Vegetal de la Universidad Nacional de Colombia sede Medellín, en donde se realizó el procedimiento de lavado, esporulación en cámara húmeda, trampeo en suelo, búsqueda de nematodos fitoparásitos, aislamiento e identificación de microorganismos y pruebas de patogenicidad. Las muestras de árboles con marchitez se identificaron siguiendo los síntomas reportados en la literatura como: marchitez generalizada, estancamiento del desarrollo, pérdida de vigor, color, brillo, amarillamiento de hojas y muerte descendente (Duque, 2011; Pérez, 2008; Tamayo, 2007; Stolzy et al., 1967).

\section{Aislamiento de microorganismos}

Las muestras de raíces colectadas se lavaron con agua, se secaron a temperatura ambiente y se seccionaron en porciones de $0.5 \mathrm{~cm}^{3}$. Luego en cabina de flujo laminar estéril se procedió a la desinfestación superficial con etanol (70\%), seguido de un lavado en agua destilada estéril e hipoclorito de sodio (3\%) cada uno por 30 s, por último se lavaron con agua destilada estéril también por 30 s. De las muestras desinfestadas se obtuvieron cortes $\left(1 \mathrm{~mm}^{3}\right)$ que fueron sembrados en medios semiselectivos para Phytophthora spp.: jugo V8 clarificado suplementado con pimaricina $(0,01 \mathrm{~g} / \mathrm{L})$, ampicilina $(0,250$ $\mathrm{g} / \mathrm{L})$, rifampicina $(0,01 \mathrm{~g} / \mathrm{L})$, pentacloronitrobenceno $(0,1$ $\mathrm{g} / \mathrm{L})$ e hymexazol $(0,05 \mathrm{~g} / \mathrm{L})$ (V8-PARPH), y jugo V8 clarificado suplementado con nistatina $(0,01 \mathrm{~g} / \mathrm{L})$, ampicilina $(0,250 \mathrm{~g} / \mathrm{L})$, rifampicina $(0,01 \mathrm{~g} / \mathrm{L})$, pentacloronitrobenceno $(0,1 \mathrm{~g} / \mathrm{L})$ e hymexazol (0,05 g/L) (V8-NARPH) (Hardham, 2005). Para hongos se utilizó papa dextrosa agar (PDA), papa dextrosa agar acidificado (PDAA), jugo V8 agar
(V8A) y para bacterias agar nutritivo suplementado con

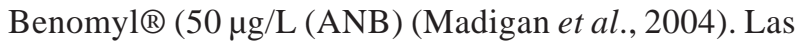
cajas de Petri sembradas se incubaron a $28^{\circ} \mathrm{C}$ por 15 días, con un fotoperíodo de 12 horas de luz y 12 de oscuridad.

\section{Trampeo de Phytophthora spp.}

Las muestras de suelo, se colocaron en un recipiente con agua para inducir la producción de unidades infectivas de Phytophthora spp. y posteriormente transferidas a frutos de aguacate y manzana para su posterior aislamiento en los medios de cultivo semi-selectivos, V8-PARPH y V8-NARPH (Hardham, 2005).

\section{Identificación taxonómica de microorganismos}

Los aislamientos obtenidos se observaron en microscopio de luz (Nikon Eclipse E200) y los agentes causales se identificaron siguiendo las claves descritas en el manual general para géneros de Barnett \& Hunter (1972), y de Erwin \& Ribeiro (1996) para las especies de Phytophthora spp.

\section{Análisis de nematodos}

La extracción de nematodos se realizó a partir de muestras de suelo seleccionadas en laboratorio con la serie de tamices de 250, 53 y $38 \mu \mathrm{m}$, éstas fueron centrifugadas durante $3 \mathrm{~min}$ a $3.800 \mathrm{rpm}$ y re-suspendidas en solución de sacarosa al $50 \%$, y después centrifugadas como se ha descrito anteriormente. El sobrenadante obtenido se pasó por el tamiz de $38 \mu \mathrm{m}$. Del material colectado se tomó una muestra para observación en microscopio de luz (Nikon Eclipse E200) (Jenkins, 1964). La identificación taxonómica se realizó usando las claves para nematodos fitoparásitos reportada por Mai \& Mullin (1996).

\section{Selección y preparación de semillas para pruebas de patogenicidad}

Semillas provenientes de plantas sanas de $P$. americana variedad guatemalteca, susceptibles a la marchitez (Ben-Ya'acov \& Frenkel, 1973), se sometieron a tratamiento hidrotérmico a $48-50^{\circ} \mathrm{C}$ por treinta minutos, luego se sumergieron en una mezcla de los fungicidas carboxín + captan durante 15 min (Tamayo, 2007). Después se removió la testa de la semilla y se realizó un corte basal de dos a cuatro mm y un corte apical de 10 a $20 \mathrm{~mm}$, para acelerar la germinación (Bernal \& Cipriano, 2008). Las semillas procesadas se sembraron en potes plásticos ( $1 \mathrm{~kg}$ de capacidad), con sustrato $(50 \%$ suelo, $25 \%$ cascarilla de arroz, $25 \%$ humus), previamente esterilizado en autoclave a 15 libras de presión y $121^{\circ} \mathrm{C}$, por dos ciclos de $1 \mathrm{~h}$. Las plantas se mantuvieron en condiciones de casa-malla, fertilizadas con la solución nutritiva Hoagland. 


\section{Inoculación delos microorganismos aislados sobre plántulas de aguacate}

Los aislamientos identificados como Cylondrocarpon sp. se inocularon siguiendo la metodología reportada por Besoain \& Piontelli, (1999) a una concentración final de $10^{6}$ propágulos $/ \mathrm{ml}$ (macroconidias y microconidias). Por su parte los aislados de Phytophthora sp., se incrementaron en papa dextrosa agar (PDA) y papa dextrosa (PD), hasta obtener una concentración de $10^{3}$ propágulos $/ \mathrm{mL}$ (esporangios), la fuente de inoculo se descargó sobre las raíces y la base del tallo de las plántulas. Para inocular los aislamientos de los géneros Verticillium sp., Fusarium sp. y Phytium sp. se aplicó una solución de agua estéril sobre las raíces y el tallo a una concentración de $10^{5}$ propágulos $/ \mathrm{mL}$ (conidias para Verticillium sp. y Fusarium sp. y esporangios para Phytium sp.). La concentración se midió en hematocitómetro y se realizo diluciones seriadas hasta alcanzar la concentración deseada.

\section{Prueba de patogenicidad para el agente abiótico asociado a la marchitez.}

Para determinar la influencia de la humedad del suelo y su correspondiente deficiencia de oxígeno, las plantas se mantuvieron a la máxima capacidad de retención de humedad del suelo (120\%), determinada previamente de la siguiente manera: se colocaron $10 \mathrm{~g}$ de suelo en mortero de porcelana con perforaciones, y este a su vez sobre un recipiente con agua, el cual permitió su movimiento por capilaridad durante 24 horas. Después la muestra de suelo se pesó (peso húmedo) y se colocó en estufa a $105{ }^{\circ} \mathrm{C}$, hasta obtener el peso constante (peso seco). El cálculo de la máxima capacidad de retención de humedad del suelo (MCRHS) se realizó mediante la siguiente fórmula: MCRHS $=[($ Peso húmedo - Peso seco) / Peso seco] x 100.

\section{Variables evaluadas}

Por cada agente causal se inocularon 5 plántulas, 30 días después de la germinación, con 5 hojas completamente expandidas (5-7 cm de altura). Las variables que se tuvieron en cuenta fueron: altura y área foliar, a los 60 días después de la inoculación (ddi) o después de someter las plantas a déficit de oxígeno, tiempo medio de aparición de síntomas (TMAS), tiempo medio de muerte de las plántulas (TMMP), sintomatología asociada y reaislamiento en los medios de cultivo antes descritos para los microorganismos identificados.

\section{Análisis estadístico}

Las mediciones morfométricas de los aislamientos se realizaron en 20 estructuras individuales. Se determinó la media y la desviación estándar para los datos obtenidos usando el programa Excel de Microsoft ${ }^{\circledR}$. Para las inoculaciones se aplicó un diseño completamente al azar con cinco repeticiones. Para las variables medidas en las pruebas de patogenicidad se evaluó la homocedasticidad y normalidad de los datos, utilizando los ensayos de Levene y Kolmogorov-Smirnov respectivamente. Después se realizó el análisis de varianza y la prueba de separación de medias con la prueba de Tukey, con un nivel de significancia del $99 \%$.

\section{RESULTADOS Y DISCUSIÓN}

\section{Incidencia de la marchitez y las labores} agronómicas asociadas al cultivo de aguacate

En la Figura 2 se muestra la incidencia de la marchitez en cada uno de los lotes evaluados, la cual fluctuó entre $10 \%$ (EL1) y $65 \%$ (DL3). Las plantas afectadas mostraron sintomatología característica asociada a la marchitez como: amarillamiento foliar, flacidez de las hojas, floración excesiva, retraso en el crecimiento, defoliación desde severa hasta leve, caída de hojas, hojas con tonalidades de color marrón que permanecen adheridas a la planta y en estados avanzados se observó muerte descendente y el sistema radicular destruido; se observaron cánceres en la base del tallo en algunas plantas adultas (Figura 3). Los arboles severamente atacados murieron, la enfermedad se presentó preferiblemente en focos localizados, en todos los estados de desarrollo de la planta desde recién establecida, hasta árbol adulto (4 años en adelante), igualmente afectando todas las variedades sembradas en los diferentes lotes.

Los tres grupos resultantes a partir de la prueba de Tukey $(P<0.05)$, se clasificaron de acuerdo al número y características de las prácticas agronómicas realizadas en el cultivo así: bueno correspondiente a la menor incidencia, medio a la incidencia intermedia y bajo a la mayor incidencia (Figura 4). Los lotes EL1, DL2, EL3 y SPL3 se ubicaron en el grupo con menor incidencia de marchitez $(10,13.3,17$ y $16.7 \%$ respectivamente). Para

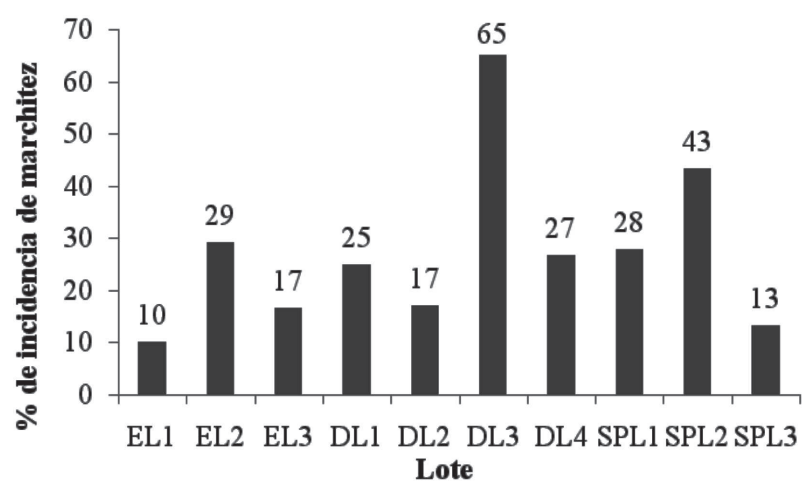

Figura 2. Porcentaje de incidencia de la marchitez del aguacate en el Norte de Antioquia-Colombia. Lotes: Donmatías: DL1, DL2, DL3 y DL4; Entreríos: EL1, EL2 y EL3 y San Pedro de Los Milagros: SPL1, SPL2 y SPL3. 

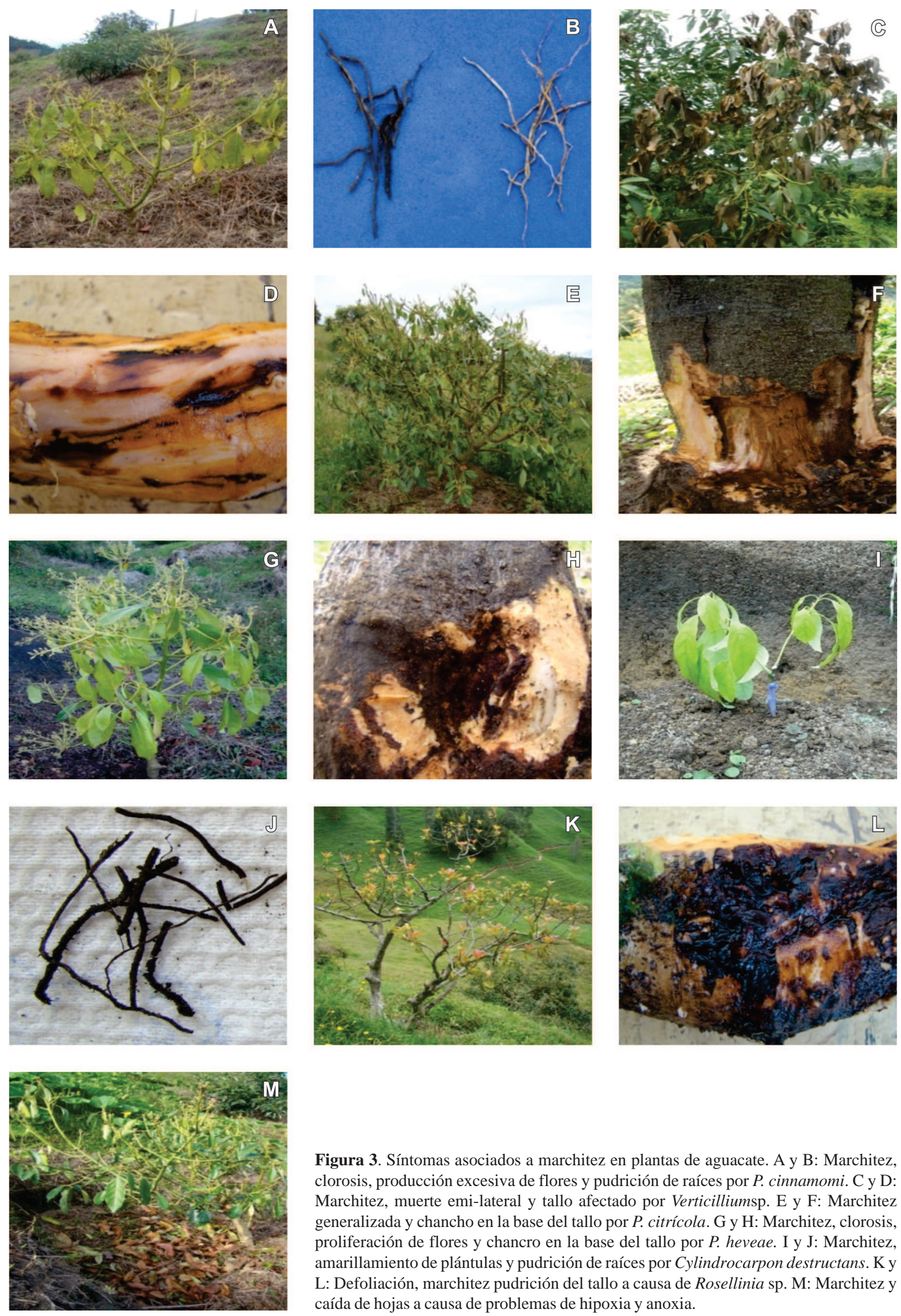

Figura 3. Síntomas asociados a marchitez en plantas de aguacate. A y B: Marchitez, clorosis, producción excesiva de flores y pudrición de raíces por $P$. cinnamomi. C y D: Marchitez, muerte emi-lateral y tallo afectado por Verticilliumsp. E y F: Marchitez generalizada y chancho en la base del tallo por P. citrícola. G y H: Marchitez, clorosis, proliferación de flores y chancro en la base del tallo por $P$. heveae. I y J: Marchitez, amarillamiento de plántulas y pudrición de raíces por Cylindrocarpon destructans. $\mathrm{K}$ y L: Defoliación, marchitez pudrición del tallo a causa de Rosellinia sp. M: Marchitez y caída de hojas a causa de problemas de hipoxia y anoxia. 
estos lotes se identificaron el mayor número de actividades apropiadas para el manejo agronómico, en general del cultivo y en particular a nivel fitosanitario. Entre estas se destacan la fertilización oportuna de acuerdo a los análisis de suelos y foliares, podas sanitarias, limpieza de herramienta de trabajo, drenajes, siembra en terrenos con pendientes mayores al $10 \%$ y aplicación de enmiendas orgánicas, entre otras.

Los lotes DL1, DL4, SPL1 y EL2, se agruparon en el nivel intermedio de incidencia $(25,26.7,27.8,29.2 \%$, respectivamente). En este grupo se observó la realización de un número menor de labores en el cultivo, principalmente la aplicación de productos químicos y la siembra en terrenos con pendiente menor al $10 \%$, en tres de cuatro lotes evaluados. Dos lotes se agruparon en el nivel más alto de incidencia de la marchitez: DL3, SPL2 (65, y 43.3\%, respectivamente). En ambos lotes se observó la siembra en terrenos con pendientes menores al $10 \%$ y la ausencia de labores agronómicas en el cultivo en el lote DL3, con incidencia del $65 \%$ y la realización de una única labor reportada en el lote SPL2 (aplicación de productos químicos).

\section{Relación entre variables de manejo agronómico e incidencia de la marchitez.}

Para la primera categoría de variables asociadas al cultivo, se identificó que las prácticas de manejo de plagas, enfermedades y arvenses $(-0.7599, P<0.0001)$ y fertilización basada en los análisis de suelo y foliares ($0.7577, P<0.0001$ ), presentaron valores altamente significativos y con relación inversa a la incidencia de síntomas de marchitez. Estos resultados sugieren que los niveles bajos de incidencia de la marchitez del aguacate están relacionados con las prácticas de manejo integrado como son el diagnóstico oportuno y control de plagas, enfermedades y arvenses, y la fertilización con base en los análisis de suelo y foliares. La asociación de las prácticas de manejo integrado de plagas, enfermedades, arvenses y fertilización, se realizan en todos los lotes que pertenecen al grupo de más baja incidencia identificado con el test de Tukey $(P<0.05)$ (Figura 4). El análisis derivado del segundo modelo para esta misma categoría, permitió incluir la variable material vegetal certificado $(-0.5240, P<0.0001)$ como significativa y con relación inversa respecto a la incidencia y la siembra en terrenos planos (pendiente menor del 10\%) (0.6339, $P<0.0001)$, con valor significativo y con relación directa con la incidencia. La significancia obtenida para este modelo indica que es recomendable comprar material de siembra certificado y sembrar en pendientes mayores al $10 \%$.

El análisis aplicado a la segunda categoría de variables relacionadas con el manejo fitosanitario de la marchitez, mostró que la poda sanitaria $(-1.0615, P<$ $0.0001)$, el reconocimiento de los síntomas $(-0.4561, P<$ 0.01 ) y el control con producto (Mancozeb-Captan-cobre) $(-0.4132, P<0.01)$, presentan relación significativa e inversa respecto a la incidencia de la marchitez, indicando que estas prácticas pueden contribuir a reducir los niveles de enfermedad en los lotes estudiados. El segundo modelo aplicado a esta categoría, permitió identificar una relación significativa e inversa para las variables desinfestación de herramienta $(-0.82272, P<0.0001)$ y uso del producto metalaxyl $(-0.41783, P<0.0001)$, para el control de los síntomas. Este análisis se puede interpretar dentro del concepto de manejo integrado del cultivo, ya que la realización correcta de todas las prácticas, para mantener el cultivo en óptimas condiciones tiene un efecto en la disminución de la incidencia. La aplicación de productos químicos se recomienda después del diagnóstico del agente causal, como citado en esta investigación, los microorganismos que pueden ocasionar la sintomatología se encuentran clasificados en grupos taxonómicos diversos, como hongos y oomycetos, sobre los cuales los productos químicos poseen eficacia y eficiencia diferentes.

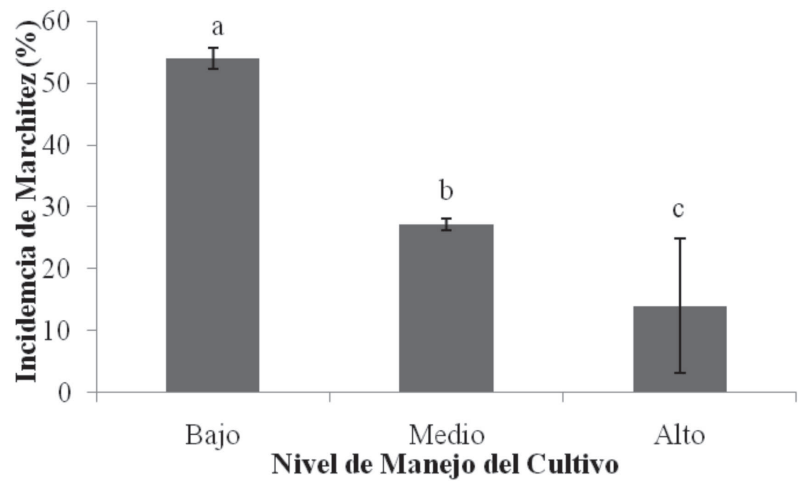

Figura 4. Rangos resultantes del manejo del cultivo y marchitez, según su desarrollo tecnológico. Lotes: Donmatías: DL1, DL2, DL3 y DL4; Entrerríos: EL1, EL2 y EL3 y San Pedro de Los Milagros: SPL1, SPL2 y SPL3. Letras distintas representan diferencias estadísticas Tukey $(P<0.05)$.

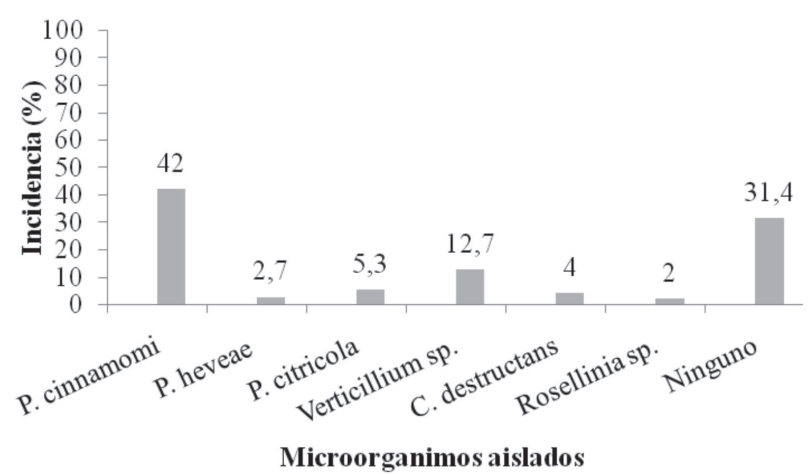

Figura 5. Incidencia de los diferentes microorganismos asociados a la marchitez en 10 lotes de la zona Norte de Antioquia. 


\section{Identificación de los microorganismos aislados de raíces y cuello de aguacate Phytophthora cinnamomi}

El $42 \%$ de aislamientos (Figura 5), se obtuvieron a partir de plantas con sintomatología de amarillamiento foliar, marchitez de hojas, retraso en el crecimiento, muerte de yemas apicales y laterales, floración excesiva, alta producción de frutos de menor calibre y maduración prematura en el árbol. Estas plantas presentaron en estados avanzados de desarrollo una escasa o nula área foliar acompañado de muerte descendente y destrucción total de las raíces secundarias (Figura 3). Los aislamientos recuperados presentaron crecimiento lento $(<$ de $10 \mathrm{~mm}$ a los 10 días) en medio V8-PARPH y V8-NARPH, ligeramente pentaloide o en roseta, coloración blanca, sin ningún tipo de pigmentación en el medio, de consistencia algodonosa; presentaron morfología micelial coraloide con frecuentes nódulos redondos, esporangios poco frecuentes en medio sólido, cuando presente no-papilado, elipsoide, base redonda y estrecha, de tamaño promedio $36.9 \pm 25 \mu \mathrm{m}$ de largo $\times 25 \pm 10 \mu \mathrm{m}$ de ancho, tipo de adhesión del esporangioesporangióforo no caduco, esporangióforo no ramificado de $3 \pm 1 \mu \mathrm{m}$, clamidosporas de aparición tardía y escasas, globosas, lisas y delgadas, terminales o intercaladas, de tamaño promedio $25.1 \pm 2 \mu \mathrm{m}$; estructuras sexuales no presentes. Los aislamientos mostraron síntomas en las plantas 21.5 ddi y produjeron la muerte de las plantas $130.3 \mathrm{ddi}$ (Tabla 1). La sintomatología en campo (Figura 3), las características macroscópicas, microscópicas y los resultados de las pruebas de patogenicidad (Figura 6 y Tabla 1) indican que estos aislamientos corresponden a $P$. cinnamomi (Pérez, 2008; Tamayo, 2007; Hardham, 2005; Erwin \& Ribeiro, 1996; Ho \& Zentmyer, 1977).

\section{Verticillium spp.}

El $12.7 \%$ de los aislamientos (Figura 5) se encontró en plantas con amarillamiento inicial generalizado, acompañado de flacidez en las hojas, detención del crecimiento de la planta, y decaimiento emi-lateral. Cuando la enfermedad avanza las hojas se tornan de una coloración café oscura permaneciendo adheridas, posteriormente empieza un proceso de muerte emi-lateral descendente, lo cual provoca la muerte completa de la planta. El corte transversal del tallo afectado mostró una coloración café típica en los haces vasculares y el sistema de raíces presentó pudrición total (Figura 3). Los aislamientos presentaron en medio PDAA micelio hialino, flocoso, tabicado y delgado, se observaron conidióforos ramificados en manojos verticilados con conidios unicelulares en los ápice, hialinas y ovoides de $6.5 \pm 2 \mu \mathrm{m}$ de largo x $3.0 \pm 1 \mu \mathrm{m}$ de ancho; al envejecer el micelio (alrededor de 20 días), las hifas se tornaron oscuras y aparecieron microesclerocios, los cuales mostraron un tamaño de $100 \pm 20 \mu \mathrm{m}$. Estos aislamientos indujeron síntomas a los 10.2 ddi y ocasionaron la muerte de las plantas a los 50.3 ddi (Tabla 1). La sintomatología (Figura 3), estructuras del patógeno y los resultados de las pruebas de patogenicidad (Figura 6 y Tabla 1), sugirieron que los aislamientos pertenecen a Verticillium sp. (Pérez, 2008; Tamayo, 2007; Zentmyer, 1984; Zentmyer, 1949).

\section{Phytophthora citrícola}

E1 5.3\% de los aislamientos (Figura 5) se procesaron a partir de árboles adultos con vigor reducido y debilitamiento progresivo, caracterizados por clorosis y caída prematura de hojas, floración excesiva, muerte de ramas y muerte de los árboles. Las raíces principales presentaron pudrición con tonalidad anaranjada o café rojizo; en la base del tallo se observaron lesiones tipo cáncer (Figura 3). El micelio presentó crecimiento lento (< de $10 \mathrm{~mm}$ a los 10 días) en medio V8-PARPH y V8-NARPH, de tipo radial, coloración blanca, sin ningún tipo de pigmentación en el medio, consistencia algodonosa, esporangios poco frecuentes, cuando presentes de semipapilados a ovoides a veces elipsoide, de tamaño promedio $45 \pm 4 \mu \mathrm{m}$ de largo x $33 \pm 12 \mu \mathrm{m}$ de ancho, adhesión del esporangio-esporangióforo no caduco, esporangióforo irregularmente ramificado. Se observó

Tabla 1. Variables evaluadas en las pruebas de patogenicidad.

\begin{tabular}{|c|c|c|c|c|c|}
\hline Agente causal implicado & TMAS $^{1}$ & TMMP $^{2}$ & Área foliar ${ }^{3}$ & Altura ${ }^{4}$ & Re-aislamiento ${ }^{5}$ \\
\hline Phytophthora cinnamomi & $21.5 \mathrm{~b}$ & $110.3 \mathrm{~b}$ & $20.1 \mathrm{c}$ & $11.9 \mathrm{~b}$ & + \\
\hline Phytophthora heveae & $35.5 \mathrm{a}$ & $105.8 \mathrm{~b}$ & $18.9 \mathrm{c}$ & $13.8 \mathrm{~b}$ & + \\
\hline Phytophthora citricola & $25.3 \mathrm{~b}$ & $115 \mathrm{~b}$ & $24.3 \mathrm{c}$ & $12.5 \mathrm{~b}$ & + \\
\hline Verticillium sp. & $10.2 \mathrm{c}$ & $50,3 \mathrm{c}$ & $14.4 \mathrm{c}$ & $11.5 \mathrm{~b}$ & + \\
\hline Cylindrocarpon destructans & $30 \mathrm{a}$ & $150.8 \mathrm{a}$ & $39.63 \mathrm{~b}$ & $14.5 \mathrm{~b}$ & + \\
\hline Déficit de oxigeno & $15 \mathrm{c}$ & $40.8 \mathrm{c}$ & $0 \mathrm{~d}$ & $8.55 \mathrm{c}$ & No aplica \\
\hline Control & - & - & $58.5 \mathrm{a}$ & $21.8 \mathrm{a}$ & No aplica \\
\hline
\end{tabular}

${ }^{1}$ Tiempo medio de aparición de síntomas de las plántulas en días después de la inoculación (ddi) o aplicación de déficit de oxígeno. ${ }^{2}$ Tiempo medio de muerte de las plántulas en ddi o de la aplicación de déficit de oxígeno. ${ }^{3}$ Área foliar en $\mathrm{cm}^{2}$, medida a los 60 ddi o de aplicación de déficit de oxígeno. ${ }^{4}$ Altura en $\mathrm{cm}$ medida a los 60 ddi o después de aplicado el déficit de oxígeno. ${ }^{5}$ re-aislamiento de agente causal en medios de cultivo. Valores con diferente letra en cada columna, presentan diferencias estadísticamente significativas según la prueba de Duncan $P<0,01$. 
producción abundante de estructuras sexuales, anteridio paragino, oogonios esféricos de pared lisa, delgados, de diámetro $28 \pm 2 \mu \mathrm{m}$, oosporas pleroticas de $20 \pm 2 \mu \mathrm{m}$ de diámetro. Cuando fueron inoculados en plantas ocasionaron síntomas 25.3 ddi y causaron la muerte de las plantas 115 ddi (Tabla 1). La sintomatología (Figura 3), características morfológicas y los resultados de las pruebas de patogenicidad (Figura 6 y Tabla 1), corresponden a las reportadas para $P$. citrícola (Erwin \& Ribeiro, 1996; Zentmyer, 1984; Zentmyer et al, 1974; Zentmyer \& Jefferson 1973; Oudemans \& Coffey 1987). Cabe resaltar que este microorganismo no ha sido reportado en Colombia causando esta patología en aguacate (Tamayo, 2007; Ciro et al., 2006; Buriticá, 1999), por lo cual este sería su primer reporte.

\section{Cylindrocarpon destructans}

El 4\% de los aislamientos (Figura 5), provenientes de un solo lote (DL1), se obtuvieron de plantas con amarillamiento generalizado, retraso en el crecimiento, flacidez de tejidos foliares, posterior defoliación y necrosis descendente, terminando en muerte de las plantas. En las raíces, se observó necrosis, sintomatología presente únicamente en plantas jóvenes (Figura 3). El aislamiento en medio PDAA, se caracterizó por rápido crecimiento (30$60 \mathrm{~mm}$, a los 10 días), micelio aéreo, textura aterciopelada o lanosa, presentando en el adverso coloración hialina de tonalidad beige, tornándose café crema; en el reverso, o diferentes tonos de amarillo, naranja y marrón, después de 7-9 días de incubación. Se observaron macroconidias en el esporodoquio, lisas, de formas rectas o ligeramente curva-
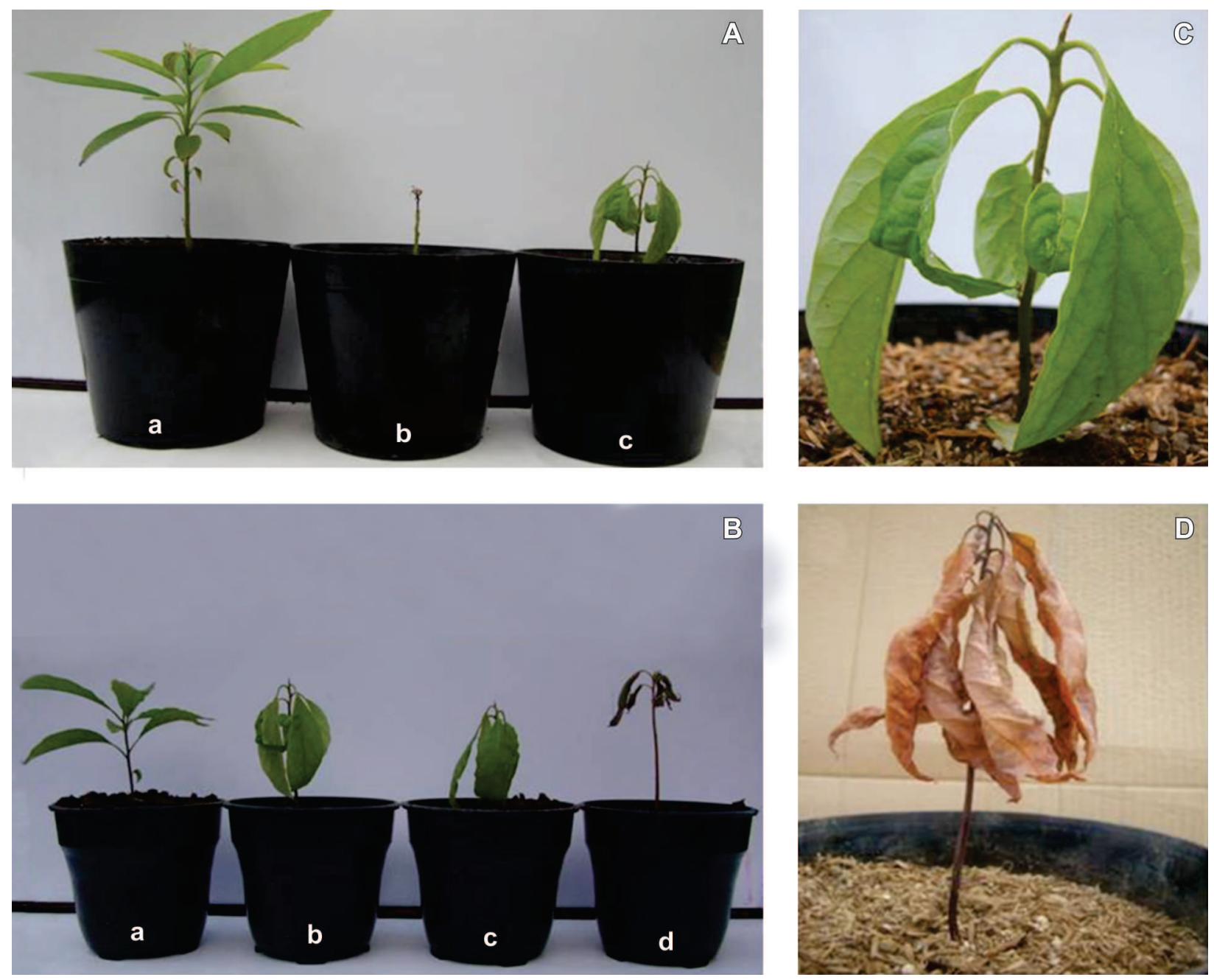

Figura 6. Pruebas de patogenicidad en aguacate (Persea americana raza Guatemalteca) para agentes causales asociados a la marchitez. A: a: planta control; b: origen abiótico de la marchitez, planta sembrada en suelo inundado, causando marchitez y perdida de las hojas; c: plántula inoculada con un aislado de $P$. cinnamomi causando retraso en el crecimiento, marchitez y pudrición de raíces B: a: planta control; b: plántula inoculada con $P$. citrícola causando marchitez y retraso en el crecimiento; c: plántula inoculada con $P$. heveae causando marchitez y retraso en el crecimiento; d: plántula inoculada con Verticullium sp., causando retraso en el crecimiento, marchitez y coloración café de las hojas. C: plántulas inoculadas con Cylindrocarpon destructans, causando síntomas de marchitez. D: síntoma típico de Verticullium spp., hojas secas que permanecen adheridas. 
das, cilíndricas a fusoides, con los extremos rectos o redondeados, base de inserción ligeramente protuberante, con presencia de 1 a 3 septos, usualmente 3, 30.2 $\pm 3.8 \mu \mathrm{m}$ de largo y $5.8 \pm 1.2 \mu \mathrm{m}$ de ancho y relación L/A de $5.2 \mu \mathrm{m}$. Se observó menor abundancia de microconidias, las cuales mostraron aspecto liso, elipsoides a cilíndricas, forma recta o ligeramente curvada, con extremos redondeados y presencia de 0 ó 1 septo, de longitud media $9.8 \pm 3.3 \mu \mathrm{m}$, ancho $2.3 \pm 0.4 \mu \mathrm{m}$ y relación L/A de $4.2 \mu \mathrm{m}$. En el medio de cultivo se identificaron clamidosporas de coloración hialina y marrón, globosas, lisas, aisladas o en cadenas o agrupadas de forma intercalar o terminal, con diámetro promedio de 9.8 $\pm 2.0 \mu \mathrm{m}$. Al inocularlos en plantas se produjeron síntomas de marchitez 30 ddi y ocasionaron la muerte de las plantas 150.8 ddi (Tabla 1). La sintomatología (Figura 3), características morfométricas y los resultados obtenidos en las pruebas de patogenicidad (Figura 6 y Tabla 1), indican que el microorganismo es Cylindrocarpon destructans (Zinss) Scholten (Booth, 1966; Samuels \& Brayford, 1990; Besoain \& Piontelli, 1999). El reporte de este patógeno afectando plántulas de aguacate en Colombia fue realizado por Ramírez \& Morales (2013), donde discuten algunos detalles de esta enfermedad.

\section{Phytophthora heveae}

El 2.7\% de los aislamientos (Figura 5) se consiguieron a partir de plantas adultas con retraso en el crecimiento, amarillamiento foliar y muerte de la planta en estados avanzados; en estas plantas la base del tallo presentó fisuras y exudación, con lesiones generalmente externas, en ocasiones extendidas al interior del xilema; al realizar cortes transversales en la corteza se observaron coloraciones rojizo-castaño (Figura 3). En medio V8PARPH y V8-NARPH presentaron micelio de crecimiento lento (< de $10 \mathrm{~mm}$ a los 10 días), ligeramente estrellado, de coloración blanca, sin ningún tipo de pigmentación en el medio, de consistencia arenosa y en algunos casos algodonosa, esporangio-papilado, frecuentemente obpiriforme, con tamaño de $43 \pm 15 \mu \mathrm{m}$ de largo $\times 27.5 \pm 9$ $\mu \mathrm{m}$ de ancho, adhesión del esporangio-esporangióforo caduco, esporangióforo irregularmente ramificado, esporangios en forma lateral, sin presencia de clamidosporas, posición anfígeno del anteridio respecto al oogonio, forma del anteridio cilíndrica, oogonio de tamaño promedio $23.7 \pm 3 \mu \mathrm{m}$, oosporas presentes, redondas, lisas y amuralladas, de $21.5 \pm 2 \mu \mathrm{m}$ de diámetro. Al inocular estos microorganismos en plántulas mostraron síntomas de marchitez 35.5 ddi y ocasionaron la muerte de las plantas 105.8 ddi (Tabla 1). La sintomatología (Figura 3 ), características morfológicas y los resultados de las pruebas de patogenicidad (Figura 6 y Tabla 1), indican que los aislamientos corresponden a $P$. heveae (Pérez, 2008; Erwin \& Ribeiro, 1996; Stamps 1978; Zentmyer et al., 1976; Zentmyer \& Jefferson 1973). Este microorganismo no ha sido reportado en Colombia causando alguna patología en aguacate (Tamayo, 2007; Ciro et al., 2006; Buriticá, 1999), por lo cual este sería el primer reporte.

\section{Rosellinia sp.}

Un 2\% de las plantas (Figura 5) presentaron marchitez generaliza, amarillamiento foliar y muerte rápida, las hojas permanecieron adheridas y adquirieron una tonalidad café oscura. Se observó reducción en el sistema de raíces, con desarrollo de masa micelial abundante de color blanco. A partir de tejido vegetal incubado en cámara húmeda a temperatura ambiente se logró realizar micromontajes; en estos se observó un micelio de forma cilíndrica, tabicado, ramificado y de color pardo oscuro, la hifas se agrupan densamente formando un estroma rojizo que cambió con el tiempo a una tonalidad más oscura, estructura comúnmente conocida cono esclerocio, a partir de estos se formaron rizomorfos. La sintomatología observada en campo y las estructuras del patógeno son similares a lo reportado en la literatura para Rosellinia sp. (Pérez, 2008; Pérez, 2006; Pérez et al., 2005). Con este microorganismo no se logró completar las pruebas de patogenicidad, por lo cual no puede afirmarse en forma concluyente, que este patógeno sea un agente causal asociado a la marchitez del aguacate.

Las especies de Fusarium sp. y Phytium sp., recuperadas en medios de cultivo no reprodujeron síntomas en las plantas inoculadas con lo cual los postulados de Koch no se lograron completar. Por otra parte a partir de las muestras colectadas en campo, no se logró identificar ninguna especie de nematodo ni de bacteria fitopatógena.

\section{Problemas abióticos asociados a la marchitez del aguacate}

En un $31.4 \%$ de las muestras (Figura 5), colectadas en campo no se obtuvieron aislamientos de microorganismos patógenos; estas plantas mostraron amarillamiento y flacidez de las hojas, estancamiento del crecimiento, muerte de yemas laterales y apicales, defoliación, pudrición total de raíces, las cuales mostraron coloración interna azul-grisácea y muerte de la planta (Figura 3). En condiciones de invernadero se logró reproducir estos síntomas en plántulas, después de 15 días de someter el suelo a un $120 \%$ de la máxima capacidad de retención de humedad (Figura 6 y Tabla 1). Este tratamiento produjo la muerte en 40.8 días después de realizado, lo cual fue más rápido que los microorganismos. Los resultados encontrados sugieren que el déficit de oxígeno, consecuencia de la inundación del suelo, induce hipoxia y anoxia, las cuales podrían representar un papel importante en los síntomas observados en campo para cultivos de aguacate (Duque, 2011; Schaffer, 2006; Stolzy et al., 1967; Valoras etal., 1964). 


\section{Pruebas de patogenicidad}

Las variables medidas en las pruebas de patogenicidad demostraron que los agentes causales implicados en la marchitez tanto bióticos como abióticos, afectan significativamente el desarrollo de las plántulas de aguacate en comparación con el control sano y presentaron síntomas de enfermedad similares a los observados en el campo (Figuras 3, 6 y Tabla 1). Los síntomas de marchitez de Verticillium sp. y por déficit de oxígeno se observaron precozmente y ocasionaron una muerte rápida. Los menores valores de altura y área foliar se observaron para el déficit de oxígeno (Figura 6 y Tabla 1). En un lugar intermedio se ubicaron las 3 especies del género Phytophthora encontradas en este estudio sin encontrarse diferencias entre ellas. El hongo Cylindrocarpon destructans afectó en menor grado el área foliar de las plantas inoculadas y presentó mayor tiempo para la aparición de síntomas y muerte de la planta. Los cinco microorganismos se re-aislaron cumpliendo con los postulados de Koch.

En este trabajo se identificaron cinco microorganismos y un factor abiótico, como causantes de los síntomas de marchitez en cultivos de aguacate. En el 50\% de las plantas con síntomas de marchitez, se identificaron 3 especies del género Phytophthora (Figura 5). La especie $P$. cinnamomi como el agente causal más frecuente asociado a la marchitez del aguacate en varios países del mundo, confirmándose esta tendencia en Colombia (Coffey, 1991; Tamayo, 2007; Ciro et al., 2006; Pérez, 2008). P. citricola y P. heveae han sido reportadas causando cáncer en la base del tallo principalmente, pero pueden además afectar las raíces principales y los frutos (Zentmyer et al, 1974; Zentmyer et al.1976; El-Hamalawi et al, 1995; Pérez, 2008). Verticillium sp. es un patógeno de importancia creciente en el país y para el cual el conocimiento es escaso a nivel mundial, puesto que las especies asociadas a la marchitez en P. americana han sido Verticillium albo atrum y Verticillium dahliae (Zentmyer 1984; Pérez, 2008). Debido a la diversidad de agentes causales que pueden ocasionar la marchitez, se hace necesaria la identificación precisa en cada caso, del agente causal, como base fundamental de un manejo integrado del problema.

En Colombia no se tiene claridad al respecto sobre el complejo marchitez, por lo que es prioritario realizar estudios que identifiquen a nivel de especie, todos los microorganismos implicados como punto de partida fundamental para un correcto diagnóstico y manejo, además de estudios más detallados sobre el agente causal de origen abiótico. Las expectativas que se han generado en torno al cultivo, las posibilidades de exportación que han abierto los tratados de libre comercio con varios países del mundo y las condiciones favorables al cultivo, son oportunidades para el desarrollo del sector agrario, sin embargo, se deben desarrollar las capacidades tecnológicas para que el cultivo del aguacate sea sostenible y competitivo en el mercado nacional e internacional.

El complejo marchitez ha sido erróneamente manejado, donde en Antioquia se ha registrado que el 68\% de los productores utilizan el control químico como única estrategia de manejo (Vásquez et al., 2011; Aproare Sat, 2009; Ciro et al., 2006). Los productos químicos dirigidos específicamente hacia el control de oomycetos no son eficaces en hongos. En este trabajo se identificaron dos especies de hongos ocasionando los síntomas de marchitez. Si se asume incorrectamente que la marchitez es ocasionada exclusivamente por microorganismos oomycetos como las especies de Phytophthora identificadas, la estrategia de manejo no es efectiva y termina con la muerte de la planta y contaminación innecesaria de suelos y aguas con productos químicos y los costos del cultivo también se incrementan, sin obtener un manejo sanitario apropiado.

\section{CONCLUSIONES}

La realización oportuna y precisa de las labores de manejo integrado del cultivo del aguacate, está directamente relacionada con la baja incidencia de marchitez. La marchitez del aguacate en la región de estudio fue ocasionada por los factores bióticos, agentes causales Phytophthora cinnamomi, P. heveae, P. citrícola, Verticillium sp., Cylindrocarpon destructans, y como factor abiótico el déficit de oxígeno en el suelo.

\section{AGRADECIMIENTOS}

A Carlos Palacios del Laboratorio de Sanidad Vegetal de la Universidad Nacional de Colombia sede Medellín, por el apoyo en el procesamiento de las muestras. También los autores expresan su agradecimiento a los productores de aguacate en las regiones evaluadas por su apoyo en esta investigación.

\section{REFERENCIAS}

Aproare Sat (2009) Línea base o diagnóstico de campo. Informativo el aguacate, 2:5-7.

Barnett H \& Hunter B (1972) Illustrated genera of inperfect fungi. $3^{\text {th }}$ edition. Minnesota, Burgess Publishing Company. 241p.

Ben-Ya'acov A \& Frenkel M (1973) Sensitivity of avocado rootstocks to Verticillium wilt. California Avocado Society Yearbook, 57:111-113.

Bernal A \& Cipriano A (2008) generalidades del cultivo de cultivo. En Tecnología para el cultivo de Aguacate. Corporación Colombiana de investigación Agropecuaria, CORPOICA, Centro de investigación La Selva, Ríonegro, Antioquia, Colombia. Manual Técnico 5. Editado por Corpoica. 241p. 
Besoain X \& Piontelli E (1999) Pudrición negra en raicillas de palto (Persea americana Mill.) por Cylindrocarpon destructans patogenicidad y aspectos epidemiológicos. Boletín Micológico, $14: 41-47$

Bonilla T, Sánchez P, Gonzales M \& Pérez M (2011) Neofusicoccum parvum y Phytium vexans: nuevos patógenos del aguacate descritos en Andalucía. En: VII Congreso Mundial del Aguacate, Cairns. Memorias, editado por avocadosource. p. $1-5$.

Booth C (1966) The genus Cylindrocarpon. Mycological Papers, 104:01-56

Buriticá PE (1999) Directorio de patógenos y enfermedades de las plantas de importancia económica en Colombia. Santafé de Bogotá, Instituto Colombiano Agropecuario. 329p.

Ciro D, Rendon K \& Navarro RA (2006) Reconocimiento de la pudrición de raíces (Phytophthora cinnamomi) en aguacate (Persea americana) en Antioquia. Revista Universidad Católica de Oriente, 22:41-51.

Coffey M (1991) Cause and diagnosis Avocado root rot. California Grower, 15:22-23.

Dann E, Forsberg L, kooke A, Pegg K, Shivas RP \& TanY (2011) The 'Cylindro' complex of avocado root pathogens. En: VII Congreso Mundial del Aguacate, Cairns. Memorias, editado por avocadosource. p.1-12.

Duque DS (2011) Asfixia radicular: estrategias de manejo en Colombia. En: VII Congreso Mundial del Aguacate, Cairns. Memorias, editado por avocadosource. p.12-24

Erwin DC \& Ribeiro OK (1996) Phytophthora Diseases Worldwide Saint Paul, The American Phytophathological Society. 562p.

El-Hamalawi ZA, Menge JA \& Guillemet FB(1995) Infection court and factors affecting the expansion of stem canker of avocado caused by Phytophthora citricola. Plant Disease, 79:384-388

Guerrero T, Nieves B, Barriga F, Aguirre S \& Coria V (2011) Recuperación de árboles de aguacate infectados con Phytophthora cinnamomi Rands bajo control biológico y químico. En: VII Congreso Mundial del Aguacate 2011, Cairns. Memorias, editado por avocadosource. p.20-43.

Hardham A (2005) Phytophthora cinnamomi. Molecular Plant Pathology, 6:589-604.

Ho H \& Zentmyer GA (1977) Morphology of Phytophthora cinnamomi. Mycology, 69:701-713.

Jenkins WR (1964) A rapid centrifugal flotation technique for separating nematodes from soil. The Plant Disease Reporter, 15:692-162.

Madigan M, Martinko J \& Parker J (2004) Blog biología de los microorganismos. $7^{\mathrm{a}}$ Ed. Madrid, Pearson Education. 1096p.

Mai WF \& Mullin PG (1996) 'Plant-parasitic nematodes- a pictorial key to genera'. Ithaca, Cornell University Press. 277p.

Mejía A (2010) Cadena Productiva del Aguacate en Colombia. Consejo Nacional del Aguacate. En: II Encuentro de la cadena productiva del aguacate, Ríonegro. Memorias, editado por Corporción Antioqueña del Aguacate. p.1:30.

Pérez RM (2008) Significant Avocado Diseases Caused by Fungi and Oomycetes. The European Journal of Plant Science and Biotechnology, 2:01-24.

Pérez RM (2006) A review of the biology and pathogenicity of Rosellinia necatrix; the cause of white root rot disease of fruit trees and otherplants. Journal of Phytopathology, 154:257266 .
Pérez RM, Zea T \& López CJ (2005) Avocado root rots in Andalucia: A review. South African Avocado Growers' Association Yearbook, 28:10-13.

Oudemans P \& Coffey MD (1987) Phytophthora citricola advances in our understanding of the disease. California Avocado Society Yearbook, 71:139-145.

Ramírez G \& Morales JG (2013) Primer informe de Cylindrocarpon destructans (Zinss) Scholten afectando plántulas de aguacate (Persea americana Mill) en Colombia. Revista Protección Vegetal, 28:27-35.

Ríos C \& Tafur R (2003) Variedades de Aguacate para el Trópico: Caso Colombia. En: World Avocado Congress, Sudafrica. Proceedings, editado por avocadosource. p.143-147.

Ruiz JC (2010) Plan de desarrollo de la Corporación Antioqueña del Aguacate. Consejo Nacional del Aguacate. En: II Encuentro de la cadena productiva del aguacate, Ríonegro. Memorias, editado por Corporción Antioqueña del Aguacate. p.10-20.

Samuels G \& Brayford D (1990) Variation in Nectria radicicola and its anamorph, Cylindrocarpon destructans. Mycological Research, 94:433-442.

Schaffer B (2006) Effects of soil oxigen deficiency on avocado (Persea americana Mill) Trees. En: Seminario Internacional Manejo del Riego y Suelo en el Cultivo del Palto, La Cruz Chile. Memorias, Editado por Instituto de Investigaciones Agropecuarias (INIA). P1- 12.

Schabenberger O \& Pierce FJ (2002) Contemporary Statistical Models for the Plant and Soil Sciences. New York, CRC Press. 300 p.

Stamps DJ (1978) CMI Descriptions of Pathogenic Fungi and Bacteria 594 Phytophthora heveae. Wallingford, CAB International. p.2.

Stolzy L, Zentmyer GA, Klotz A \& Labanauskas C (1967) Oxygen diffusion, water, and Phytophthoracinnamomi in root decay and nutrition of avocados. American Society for Horticultural Science, 90:67-76.

Tamayo PJ (2007) Enfermedades del aguacate. Revista Politécnica, 4:52-71.

Valoras N, Letey J, Stolzy L \& Frolich F (1964) The Oxygen Requirements for Root Growth of Three Avocado Varieties. American Society for Horticultural Science, 85:172-178.

Vásquez L, Ríos G, Londoño M \& Torres M (2011) Caracterización biofísica y socioeconómica del sistema de producción de aguacate cv Hass en los departamentos de Antioquia, Caldas, Risaralda y Quindío. Corporación Colombiana de investigación CORPOICA. Editado por Corpoica. 54 p.

Vitale A, Aiello D, Guarnaccia V, Perrone G, Stea G \& Polizzi G (2012) First Report of Root Rot Caused by Ilyonectria (=Neonectria) macrodidyma on Avocado (Persea americana) in Italy. Journal of Phytopatholy, 160:156-159.

Zentmyer GA (1949) Verticillium wilt of avocado. Phytopathology, 39:677-682.

Zentmyer GA (1984) Avocado diseases.Tropical Pest Management, $30: 388-400$.

Zentmyer GA, Jefferson L, Hickman CJ \& Ko YC (1974) Studies of Phytophthora citricola isolated from Persea americana. Mycologia, 66:830-845.

Zentmyer GA \& Jefferson L (1973) Another species of Phytophthora on avocado in California. California Avocado Society Yearbook, 56:125-129.

Zentmyer GA, Klure LJ \& Neal E (1976) Trunk canker of avocado caused by Phytophhtora heveae. California Avocado Society Yearbook, 60:169-170. 